\title{
Adaptive Zero-Padding OFDM over Frequency-Selective Multipath Channels
}

\author{
Neng Wang \\ Department of Electrical and Computer Engineering, Queen's University, Kingston, Ontario, Canada K7L 3N6 \\ Email:nwang@ee.queensu.ca
}

Steven D. Blostein

Department of Electrical and Computer Engineering, Queen's University, Kingston, Ontario, Canada K7L 3N6

Email:sdb@ee.queensu.ca

Received 28 February 2003; Revised 22 August 2003

\begin{abstract}
We present a novel bandwidth (BW) efficient orthogonal frequency division multiplexing (OFDM) scheme with adaptive zeropadding (AZP-OFDM) for wireless transmission. Redundancy issues in OFDM based on cyclic prefix (CP), zero-padding (ZP), as well as no guard interval (NGI) systems are analyzed. A novel system design criterion based on the channel matrix condition is studied and applied to the design of an AZP-OFDM system. Simulation results have shown that the proposed AZP-OFDM offers performance similar to that of CP-OFDM, complexity similar to that of ZP-OFDM, with BW efficiency higher than that of both CP- and ZP-OFDM in channels with small to moderate delay spread. In channels with large delay spread, AZP scheme adaptively maintains high performance at the expense of BW efficiency. Essentially, AZP-OFDM offers a more flexible tradeoff between symbol recovery, BW efficiency, and complexity.
\end{abstract}

Keywords and phrases: bandwidth efficiency, cyclic prefix, equalization, multipath channels, orthogonal frequency division multiplexing (OFDM), zero-padding.

\section{INTRODUCTION}

Orthogonal frequency division multiplexing (OFDM) has been receiving growing interest in recent years and has been adopted in many standards. For example, OFDM has been chosen as a solution for digital audio and video broadcasting (DAB and DVB) in Europe, and applied for high-speed digital subscriber line (DSL) modems over twisted pairs (ADSL, HDSL, and VDSL). Recently, it has also been proposed for digital cable television systems and adopted in new standards for wireless local area networks (wireless LAN) in North America (IEEE 802.11a), in Europe (HIPERLAN/2), and in Asia (MMAC) [1].

All standard OFDM systems are based on a cyclic prefix (CP) to eliminate interblock interference (IBI) between successive blocks. A CP of length no less than the channel order is inserted per transmitted block. Discarding the CP at the receiver not only suppresses IBI, but also converts the linear channel convolution into a circular one, which facilitates the diagonalization of the channel matrix, and makes single-tap equalization using scalar division possible [1]. An obvious problem in CP-OFDM systems is that the transmitted symbols cannot be recovered when some channel zeros are located on subcarriers. Recently, it has been proposed in $[1,2]$ to replace $\mathrm{CP}$ insertion by zero-padding $(\mathrm{ZP})$ at the end of the block of symbols to be transmitted. The padded zeros deterministically suppress the IBI but lead to a larger number of observed samples. That way, the transmitted symbols can always be retrieved regardless of the channel zero locations [1]. Note that since the number of zeros required to cancel IBI equals the CP length, ZP- and CP-OFDM transmissions have the same bandwidth (BW) efficiency. For some existing OFDM systems, such as HIPERLAN/2 and DAB physical layer, the length of the $\mathrm{CP}$ is chosen to be $1 / 4$ of the block size, which results in a BW efficiency of $80 \%$.

Approaches that have been proposed to increase the BW efficiency in OFDM systems include $[3,4,5]$. In [3], an OFDM system that does not use a guard interval (referred to here as NGI-OFDM) was proposed. Because equalization in such a system may be ill-conditioned, a Moore-Penrose pseudoinverse of the channel matrix has to be performed. A partial response (PR) OFDM transmission scheme has been proposed in [4] that employs a smooth window function. In [5], a vector OFDM scheme was proposed which performs CP insertion after blocking of conventional OFDM data blocks so that the average overhead due to the guard interval is 


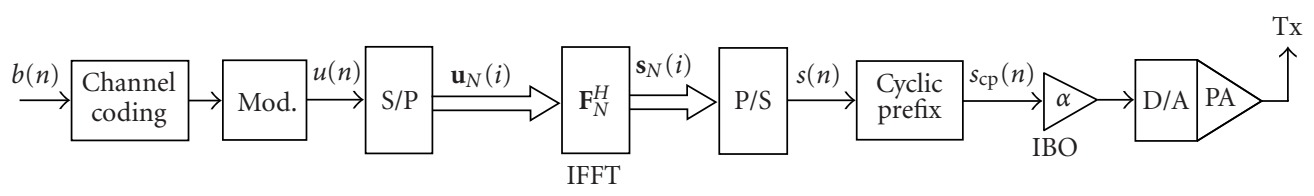

Figure 1: Block diagram of CP-OFDM transmitter.

decreased. In wireline applications, channel impulse response shortening techniques have been developed so that the length of the guard interval can be reduced [6]. Application in wireless systems may be very difficult due to the time-varying characteristic of wireless channels.

In this paper, we propose a novel OFDM transmission scheme with adaptive zero-padding (AZP-OFDM). We first examine the redundancy introduced in CP- and ZP-OFDM as well as OFDM without using a guard interval. The reduction of redundancy is then investigated and a novel system design criterion based on channel matrix condition is introduced. Using this criterion, a BW efficient AZPOFDM scheme is developed. Complexity issues are also addressed in system design, which results in more flexibility in that one can make tradeoff among performance, efficiency, and complexity. Generally multicarrier transmission suffers from problems including high peak-to-average power ratio (PAPR) ([7]). By reducing the redundancy, the proposed AZP scheme achieves improved signal-to-noise ratio (SNR) compared to that of ZP-OFDM, which is an added benefit besides increased BW efficiency. In channels with large delay spread, simulation results show that the proposed AZPOFDM offers better performance than those of both CP- and ZP-OFDM with fixed guard length.

This paper is organized as follows: a brief description of CP- and ZP-OFDM as well as NGI-OFDM are provided in Section 2. Section 3 addresses redundancy issues in these schemes. In Section 4, we first propose a system design criterion based on channel matrix condition. The application to OFDM to tradeoff BW efficiency and performance leads to our proposed AZP-OFDM scheme. A modification to lower equalization complexity is also discussed, along with an algorithm for choosing the key parameter. Simulation results and some further discussions in the context of HIPERLAN/2 channel models as well as channels with exponential power delay profiles are provided in Section 5.

\section{SYSTEM MODEL}

\subsection{CP-OFDM}

A baseband discrete-time equivalent model of a CP-OFDM transmitter is shown in Figure 1. A serial stream of information bits $b(n)$ is first passed through an error-control encoder, then serial-to-parallel (S/P) converted into data blocks of size $N, \mathbf{u}_{N}(i) \stackrel{\text { def }}{=}[u(i N), u(i N+1), \ldots, u(i N+N-1)]^{T}$. Performing multicarrier modulation via inverse fast Fourier transform (IFFT), we obtain

$$
\mathbf{s}_{N}(i)=\mathbf{F}_{N}^{H} \mathbf{u}_{N}(i),
$$

where $\mathbf{F}_{N}$ is the $N \times N$ FFT matrix with $\left[\mathbf{F}_{N}\right]_{n, k}=$ $(1 / \sqrt{N}) e^{-j 2 \pi n k / N}$, and $(\cdot)^{H}$ denotes Hermitian transpose. A length $G \mathrm{CP}$ is then inserted between each block, and the resulting redundant $i$ th block $\mathbf{s}_{\mathrm{cp}}(i)$ of length $P=N+$ $G$ is parallel-to-serial (P/S) converted into time-domain samples $s_{\mathrm{cp}}(n)$ and sent sequentially through the channel. The input back-off (IBO) and nonlinear distortion introduced by the power amplifier (PA) will be discussed in Section 5. The multipath propagation channel can be modeled as a finite impulse response (FIR) filter with tap vector $\mathbf{h} \stackrel{\text { def }}{=}\left[h_{0}, h_{1}, \ldots, h_{L}, 0, \ldots, 0\right]^{T}$ and additive white Gaussian noise (AWGN) $\eta(n) \sim N\left(0, \sigma^{2}\right)$. This baseband discretetime equivalent model combines the effects of the spectralshaping, sampling the continuous-time channel, and the receive-filter.

We assume perfect symbol and block synchronization. If the length of the CP, $G$, is no less than the channel model order $L$, after discarding the first $G$ entries corresponding to the $\mathrm{CP}$, we obtain ${ }^{1}$

$$
\mathbf{r}_{\mathrm{cp}}(i)=\widetilde{\mathbf{H}} \mathbf{s}_{N}(i)+\boldsymbol{\eta}_{N}(i),
$$

where $\widetilde{\mathbf{H}}$ is the $N \times N$ circulant matrix [9] with first column $\left[h_{0}, \ldots, h_{L}, 0, \ldots, 0\right]^{T}$; and $\boldsymbol{\eta}_{N}(i)$ is the $N \times 1$ AWGN vector. After demodulation by FFT, we obtain

$$
\begin{aligned}
\mathbf{y}_{\mathrm{cp}}(i) & =\mathbf{F}_{N} \widetilde{\mathbf{H}} \mathbf{F}_{N}^{H} \mathbf{u}(i)+\mathbf{F}_{N} \boldsymbol{\eta}_{N}(i) \\
& =\mathbf{D}_{N}(H) \mathbf{u}(i)+\mathbf{n}(i),
\end{aligned}
$$

where $\mathbf{D}_{N}(H)=\operatorname{diag}(H(0), \ldots, H(N-1))$ with $H(k)=$ $\sum_{l=0}^{L} h_{l} e^{-j 2 \pi k l / N}$ denoting the channel frequency response on the $k$ th subcarrier, and $\mathbf{n}(i)=\mathbf{F}_{N} \boldsymbol{\eta}_{N}(i)$ is the FFT-processed noise vector. Note that in (3), we use the well-known property that a circulant matrix can be diagonalized by pre- and postmultiplication by FFT and IFFT matrices. This property facilities simple one-tap equalization for CP-OFDM.

\subsection{ZP-OFDM}

ZP-OFDM [2] transmission differs from CP-OFDM only in that instead of $C P$ insertion, a guard interval of $G$ zeros is padded at the end of each block. Assuming $G \geq L$, the received block is now given by

$$
\mathbf{r}_{\mathrm{zp}}(i)=\overline{\mathbf{H}} \mathbf{s}_{N}(i)+\boldsymbol{\eta}_{P}(i),
$$

\footnotetext{
${ }^{1}$ Note that if $G<L,(2)$ is not valid due to residual IBI. For a general signal model with IBI, see [8].
} 
where $\overline{\mathbf{H}}$ is a $P \times N$ tall Toeplitz matrix with first column $\left[h_{0}, \ldots, h_{L}, 0, \ldots, 0\right]^{T}$. Zero-forcing $(\mathrm{ZF})$ or minimum mean squared error (MMSE) equalization can be performed at the receiver [1]. However, direct ZF or MMSE equalization requires the inversion of a $P \times P$ or $N \times N$ matrix, respectively. The computation can be lowered with fast algorithms based on the FFT [10].

\subsection{NGI-OFDM}

NGI-OFDM for frequency-selective channels was proposed in [3] to achieve maximal BW efficiency. NGI-OFDM has the same transmitter structure as CP-OFDM except that there is NGI inserted and removed. The IBI due to time dispersion of the channel is combated by ISI cancellation as in [8]. After IBI cancellation, the "IBI-free" received block symbol is given by

$$
\mathbf{r}_{\text {ngi }}(i)=\mathbf{H}_{N} \mathbf{s}_{N}(i)+\boldsymbol{\eta}_{N}(i)
$$

where $\mathbf{H}_{N}$ is an $N \times N$ lower triangular Toeplitz matrix with first column $\left[h_{0}, \ldots, h_{L}, 0, \ldots, 0\right]^{T}$, which is not circulant and cannot be diagonalized with FFT and IFFT matrices. Therefore, intercarrier interference (ICI) has been introduced and must be mitigated for symbol detection by solving (5). To avoid stability problems with matrix inversion for a probably ill-conditioned channel matrix, Moore-Penrose pseudoinversion [9] is employed in [3]. However, the ICI cancellation algorithm in [3] does not take advantage of the lower triangular Toeplitz structure of the channel matrix $\mathbf{H}_{N}$. Furthermore, from our analysis (Section 3) and simulations (Section 5) in the context of standard HIPERLAN/2 channel models, the system performance of NGI-OFDM over hostile wireless propagation channels is not acceptable.

\section{REDUNDANCY ISSUES IN OFDM TRANSMISSION}

Note that the length of the guard interval in ZP-OFDM is the same as that in CP-OFDM. Both have the same BW efficiency $N /(N+G)$. Typically, $G=N / 4$ (e.g., in DAB and HIPER$\mathrm{LAN} / 2$ ), which results in a BW efficiency of $80 \%$. The insertion of CP or trailing zeros introduces redundancy in OFDM transmission and decreases the system throughput, which is unattractive in high-rate communications. We now discuss whether such redundancy is necessary for OFDM systems.

\subsection{CP- and ZP-OFDM}

As described above, CP-OFDM uses redundancy in the CP to cancel IBI and transform the linear convolutional channel into a circular one (see (2)), which makes simple onetap equalization possible (see (3)). One main problem in CPOFDM is that the transmitted symbols cannot be recovered when some subcarriers encounter channel zeros even in the absence of noise [1]. Moreover, if a channel zero is located close to the DFT grid $\left(\left|H\left(k_{0}\right)\right| \ll 1\right)$, then equalization will suffer from a "noise-enhancement" problem. Therefore, $C P$ OFDM offers simple equalization without guaranteed symbol recovery.
In ZP-OFDM, redundancy is introduced in terms of $G$ trailing zeros, which not only cancels IBI but also guarantees symbol recovery. This can be shown as follows. Consider the noise-free received block in (4). Taking $\mathbb{Z}$-transform

$$
R_{\mathrm{zp}}(z)=H(z) S(z)
$$

ZF equalization for ZP-OFDM turns out to be a deconvolution procedure. The DFT of $\mathbf{r}_{\mathrm{zp}}(i)$ is obtained by sampling its Fourier transform:

$$
R_{\mathrm{zp}}\left(e^{j \omega}\right)=\left.R_{\mathrm{zp}}(z)\right|_{z=e^{j \omega}}=H\left(e^{j \omega}\right) S\left(e^{j \omega}\right) .
$$

To avoid aliasing, at least $P(\geq N+L)$ samples should be evaluated $[11]:^{2}$

$$
\begin{aligned}
& R_{\mathrm{zp}}\left(e^{j(2 k \pi / P)}\right) \\
& \quad=H\left(e^{j(2 k \pi / P)}\right) S\left(e^{j(2 k \pi / P)}\right), \quad k=0,1, \ldots, P-1 ;
\end{aligned}
$$

that is, there are $P$ virtual subcarriers compared to $\mathrm{CP}$ OFDM, where the number of subcarriers is $N$. Heuristically, more virtual subcarriers offer diversity and immunity to channel nulls. In other words, robustness to channel nulls in ZP-OFDM is achieved by means of using $P$ virtual subcarriers. The price paid for this benefit is increased equalization complexity [10]. Therefore, ZP-OFDM guarantees symbol recovery with increased complexity.

\subsection{NGI-OFDM}

Equalization in NGI-OFDM is achieved by solving the linear systems of equations in (5). The perturbation analysis of direct methods for solving such linear equations (see, e.g., $[12,13])$ is summarized in the following theorem. ${ }^{3}$

Theorem 1 (see [12, Theorem 7.7.4]). Consider the linear system $\mathbf{A x}=\mathbf{b}$, where the matrix $\mathbf{A} \in \mathbf{R}^{n \times n}$ is nonsingular. Let $(\mathbf{A}+\delta \mathbf{A})(\mathbf{x}+\delta \mathbf{x})=\mathbf{b}+\delta \mathbf{b}$ be a perturbed system and assume that

$$
\varepsilon \stackrel{\text { def }}{=}\left\|\mathbf{A}^{-1}\right\| \cdot\|\delta \mathbf{A}\|=\kappa(\mathbf{A}) \frac{\|\delta \mathbf{A}\|}{\|\mathbf{A}\|}<1 .
$$

Then $(\mathbf{A}+\delta \mathbf{A})$ is nonsingular and the norm of the perturbation $\delta \mathbf{x}$ is bounded by

$$
\frac{\|\delta \mathbf{x}\|}{\|\mathbf{x}\|} \leq \frac{\kappa(\mathbf{A})}{1-\varepsilon}\left(\frac{\|\delta \mathbf{A}\|}{\|\mathbf{A}\|}+\frac{\|\delta \mathbf{b}\|}{\|\mathbf{b}\|}\right),
$$

where $\kappa(\mathbf{A}) \stackrel{\text { def }}{=}\|\mathbf{A}\| \cdot\left\|\mathbf{A}^{-1}\right\|$ is the condition number with respect to the problem of matrix inversion.

We note that $\kappa(\mathbf{A})$ depends on the choice of matrix norm, for example, if the 2 -norm is chosen, $\kappa_{2}(\mathbf{A})=\sigma_{\max } / \sigma_{\min }$ with

\footnotetext{
${ }^{2}$ Note that this has also been used in [10] for fast ZF and MMSE equalization of ZP-OFDM.

${ }^{3}$ Note that this theorem holds for any consistent vector and matrix norms, and is not restricted to the 2-norm [12].
} 
$\sigma_{\max }$ and $\sigma_{\min }$ denote, respectively, the maximum and minimum singular values of $\mathbf{A}$ [12]. Consider the equalization problem of NGI-OFDM in (5). For simplicity of notation, we drop the indices and subscripts,

$$
\mathbf{r}=\mathrm{Hs}+\boldsymbol{\eta} .
$$

Assuming no channel mismatch, that is, $\delta \mathbf{H}=0$, the condition in (9) is satisfied since $\varepsilon=\left\|\mathbf{H}^{-1}\right\| \cdot\|\delta \mathbf{H}\|=0$. Define the SNR at the input and output of the equalizer as, respectively,

$$
\gamma_{\text {in }} \stackrel{\text { def }}{=} \frac{\|\mathbf{H s}\|^{2}}{\|\boldsymbol{\eta}\|^{2}}, \quad \gamma_{\text {out }} \stackrel{\text { def }}{=} \frac{\|\boldsymbol{s}\|^{2}}{\|\hat{\boldsymbol{s}}-\mathbf{s}\|^{2}} \equiv \frac{\|\mathbf{s}\|^{2}}{\left\|\mathbf{H}^{\dagger} \mathbf{r}-\mathbf{s}\right\|^{2}},
$$

where $\hat{\mathbf{s}}=\mathbf{H}^{\dagger} \mathbf{r}$ is the output of the equalizer, and $(\cdot)^{\dagger}$ denotes the pseudoinverse. We have the following corollary.

Corollary 2. The relative estimation error and the SNR at the output of the equalizer of NGI-OFDM are bounded by, respectively,

$$
\begin{aligned}
\frac{\|\hat{\boldsymbol{s}}-\boldsymbol{s}\|}{\|\mathbf{s}\|} & \leq \kappa(\mathbf{H}) \frac{\|\boldsymbol{\eta}\|}{\|\mathbf{H} \boldsymbol{s}\|}, \\
\gamma_{\text {out }} & \geq \frac{\gamma_{\text {in }}}{\kappa^{2}(\mathbf{H})} .
\end{aligned}
$$

Since multicarrier demodulation with FFT is a unitary transform, the SNR remains the same at the input of decision devices, which determines system performance. For example, for QPSK modulation, the symbol error rate (SER) is given by [14]

$$
P_{s}=2 Q\left(\sqrt{\gamma_{\text {out }}}\right)\left[1-\frac{1}{2} Q\left(\sqrt{\gamma_{\text {out }}}\right)\right]
$$

where $Q(x) \stackrel{\text { def }}{=}(1 / \sqrt{2 \pi}) \int_{x}^{\infty} e^{-t^{2} / 2} d t$.

An example of the equalizer output SNR, $\gamma_{\text {out }}$, with ZF equalization in HIPERLAN/2 channel A [15] and the bound from Corollary 2 is shown in Figure 2. We can see that the bound is not tight. However, from Figure 2, we can see that this bound is proportional to the mean square estimation error (MSEE). Therefore, the lower bound in (14) may serve as a predictor for $\gamma_{\text {out }}$. Figure 3 shows an example of the condition number of NGI-OFDM channel matrix varying with time in HIPERLAN/2 channel A with a terminal speed $v=3(\mathrm{~m} / \mathrm{s})$. As a comparison, those of CP- and ZP-OFDM are also shown. From Figure 3, we can see that the condition number of NGI-OFDM is usually very large, which makes equalization in (5) an ill-conditioned problem, and moreover causes unacceptable performance in NGI-OFDM, as will be shown in Section 5 .

We note that equalization in CP-OFDM also requires solving linear equations (2). So the above perturbation analysis for NGI-OFDM applies. For ZP-OFDM, the system is overdetermined and the above analysis has to be modified, as detailed in Section 4. From Figure 3, we see that the condition number of the CP-OFDM channel matrix is usually much smaller compared to that of NGI-OFDM, which explains why CP-OFDM offers better performance than NGIOFDM. This can also be shown from a signals-and-systems

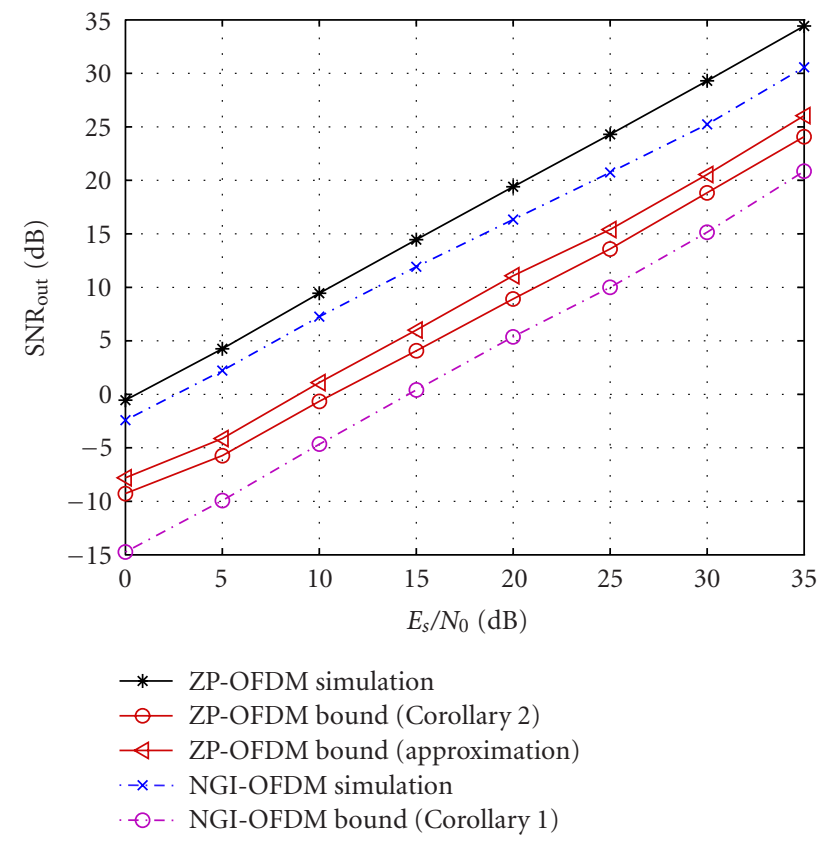

FIGURE 2: Equalizer output SNR and bounds for ZF equalization in HIPERLAN/2 channel A.

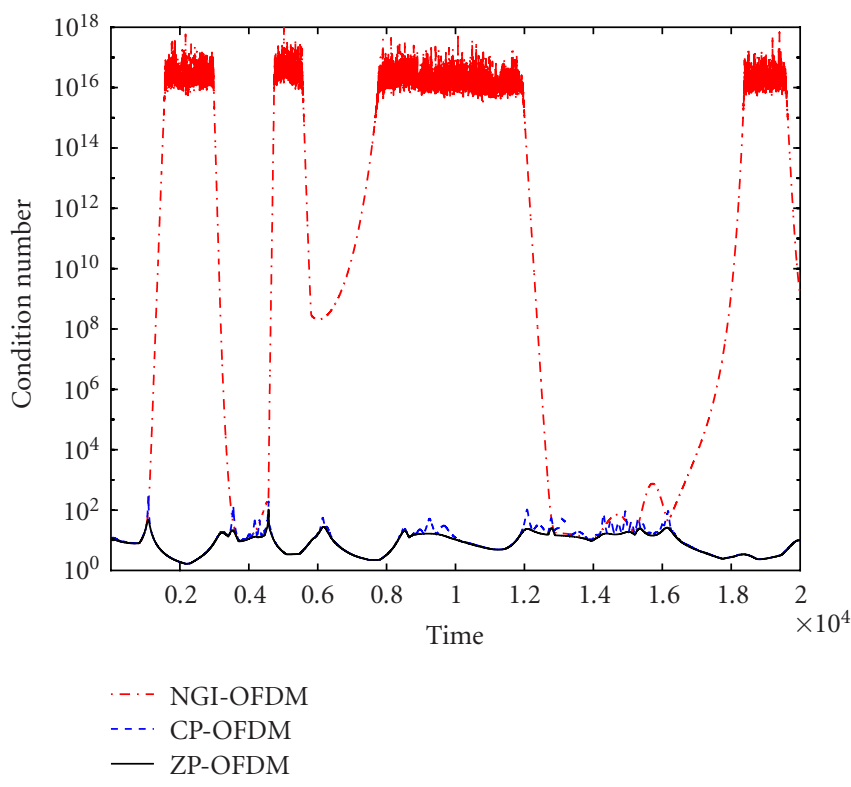

FIGURE 3: Condition number of NGI-OFDM channel matrix variation with time in HIPERLAN/2 channel A with terminal speed $v=3(\mathrm{~m} / \mathrm{s})$.

viewpoint. From the received signal models in (4) and (5), we know that the NGI signal is a length- $N$ rectangularly windowed version of ZP-OFDM, which suffers from Gibbs phenomenon [16]. In the Z-transform domain, $R_{\mathrm{ngi}}(z)$ is obtained by discarding those items with power less than $1-N$. Consider the system identification problem to find an equivalent convolutional channel for NGI-OFDM, that is, to find 


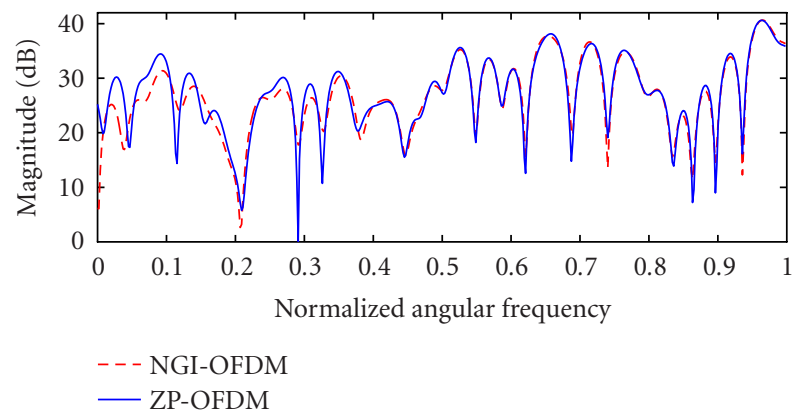

(a)

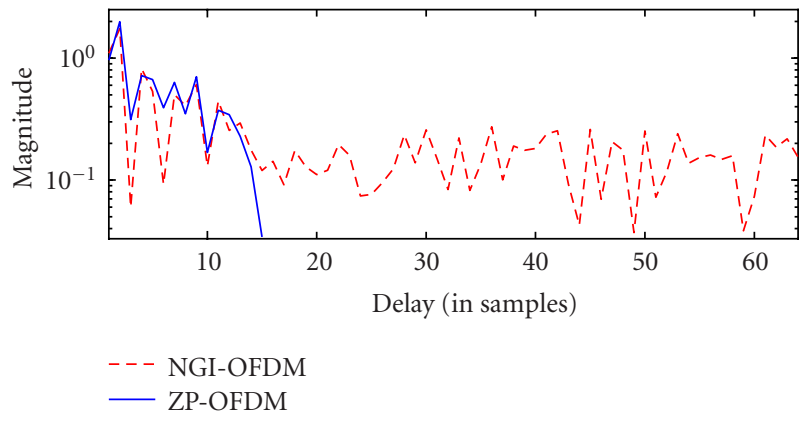

(b)

Figure 4: The Gibbs phenomenon and channel impulse response lengthening effect in NGI-OFDM $(N=64, L=14)$. (a) Magnitude response of the received signal; (b) channel impulse response.

$\hat{H}(z)$ such that

$$
R_{\text {ngi }}(z)=\hat{H}(z) S(z) .
$$

Since $S(x)$ divides $R_{\text {zp }}(z)$, it cannot divide $R_{\text {ngi }}(z)$. Therefore, the effective channel impulse response $\hat{h}(l)=\mathcal{Z}^{-1}\{\hat{H}(z)\}$ is lengthened. Figure 4 shows an example of the channel impulse response lengthening effect in NGI-OFDM systems. Now that the effective channel order is larger than the original one, $L$, there are more channel nulls and the possibility for subcarriers encounter channel nulls is also increased.

Compared with CP- and ZP-based schemes, NGI-OFDM offers high BW efficiency, but suffers from inferior performance and high complexity, as will be discussed. We conclude that redundancy is required for OFDM transmission over wireless channels. Therefore, it is meaningful to investigate introducing redundancy in a "better" way, or, in other words, to find better tradeoffs among efficiency, performance, and complexity.

\section{PROPOSED AZP-OFDM SCHEME}

To increase the BW efficiency of ZP-OFDM systems, redundancy introduced in transmission must be reduced. In fact, from the guaranteed symbol recovery property of ZP-OFDM discussed in Section 3, we find that the necessary amount of redundancy is $L$. Padding more zeros does not affect the system performance and only results in lower BW efficiency. On the other hand, if we loosen the constraint on symbol recovery slightly by padding less zeros, higher BW efficiency may be achieved. This does not mean a loss of symbol recovery capability, but a tradeoff between efficiency and performance, as will be clear in Section 4.1. Tradeoffs including complexity issues will be discussed in Section 4.2.

\subsection{AZP-OFDM scheme}

Consider the signal model in ZP-OFDM. Rewrite (4) as in (11). Equalization in ZP-OFDM requires solving an overdetermined system, which can be easily treated as a full-rank least squares (LS) problem $[9,12,13]$. The sensitivity of the LS problem is well measured by the condition of channel ma- trix $\mathbf{H}$ with respect to the 2-norm $\kappa_{2}(\mathbf{H}) \stackrel{\text { def }}{=} \sigma_{1} / \sigma_{N}$, where $\sigma_{1}$ and $\sigma_{N}$ denote, respectively, the maximum and minimum singular values of $\mathbf{H}$.

Theorem 3 (see [13, Theorem 3.4]). Suppose that $\mathbf{A}$ is $m$-by$n$ with $m \geq n$ and has full rank. Suppose that $\mathbf{x}$ minimizes $\|\mathbf{A x}-\mathbf{b}\|$. Let $\boldsymbol{\xi}=\mathbf{A} \mathbf{x}-\mathbf{b}$ be the residual. Let $\hat{\mathbf{x}}$ minimize $\|(\mathbf{A}+\delta \mathbf{A}) \hat{\mathbf{x}}-(\mathbf{b}+\delta \mathbf{b})\|$. Assume

$$
\epsilon \stackrel{\text { def }}{=} \max \left(\frac{\|\delta \mathbf{A}\|}{\|\mathbf{A}\|}, \frac{\|\delta \mathbf{b}\|}{\|\mathbf{b}\|}\right)<\frac{1}{\kappa_{2}(\mathbf{A})} \equiv \frac{\sigma_{n}}{\sigma_{1}} .
$$

Then

$$
\begin{aligned}
\frac{\|\hat{\mathbf{x}}-\mathbf{x}\|}{\|\mathbf{x}\|} & \leq \epsilon \cdot\left(\frac{2 \kappa_{2}(\mathbf{A})}{\cos (\theta)}+\tan (\theta)\left(\kappa_{2}(\mathbf{A})\right)^{2}\right)+\mathcal{O}\left(\epsilon^{2}\right) \\
& \equiv \epsilon \cdot \kappa_{\mathrm{LS}}+\mathcal{O}\left(\epsilon^{2}\right),
\end{aligned}
$$

where $\theta$ is defined by $\sin (\theta) \stackrel{\text { def }}{=}\|\boldsymbol{\xi}\| /\|\mathbf{b}\|$, that is, $\theta$ is the angle between the vectors $\mathbf{b}$ and $\mathbf{A} \mathbf{x}$ and measures whether the residual norm $\|\boldsymbol{\xi}\|$ is large (near $\|\mathbf{b}\|$ ) or small (near 0$). \kappa_{\mathrm{LS}}$ is the condition number of the LS problem.

Note that the assumption $\epsilon \cdot \kappa_{2}(\mathbf{A})<1$ guarantees that $\mathbf{A}+$ $\delta \mathbf{A}$ has full rank so that $\hat{\mathbf{x}}$ is uniquely determined. This bound can be interpreted as [13]: if $\theta$ is 0 or very small, then the residual is small and the effective condition number is about $2 \kappa_{2}(\mathbf{A})$, much like ordinary linear equation solving; if $\theta$ is not small but not close to $\pi / 2$, the residual is moderately large, and then the effective condition number can be much larger: $\kappa_{2}^{2}(\mathbf{A})$; if $\theta$ is close to $\pi / 2$, the true solution is nearly zero, then the effective condition number becomes unbounded even if $\kappa_{2}(\mathbf{A})$ is small.

For our equalization problem in (11), if we assume no channel mismatch, that is, $\delta \mathbf{H}=0$; then the residual $\xi \stackrel{\text { def }}{=}$ Hs $-(\mathbf{r}-\boldsymbol{\eta})=0$, so $\sin (\theta)=0, \tan (\theta)=0$. Define $\epsilon \stackrel{\text { def }}{=}$ $\|\boldsymbol{\eta}\| /\|\mathbf{H s}\|$ and the SNR at the input of the equalizer $\gamma_{\text {in }} \stackrel{\text { def }}{=}$ $\|\mathbf{r}\|^{2} /\|\boldsymbol{\eta}\|^{2}=1 / \epsilon^{2}$. Condition (17) is equivalent to

$$
\gamma_{\text {in }}>\kappa_{2}^{2}(\mathbf{H})
$$




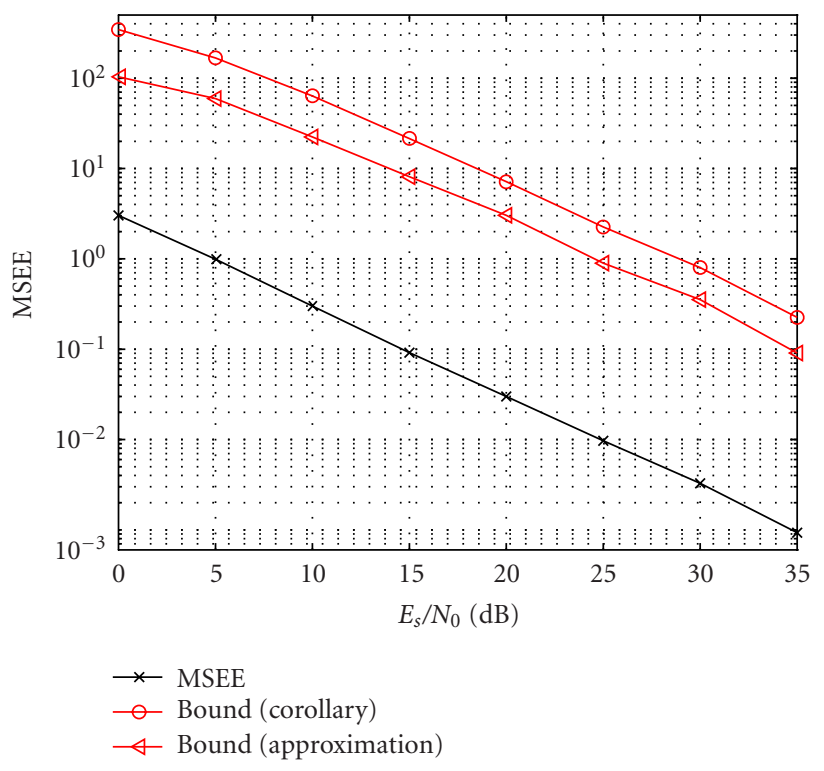

Figure 5: MSEE and bounds for ZF equalization in HIPERLAN/2 channel A.

which may be violated, but (18) still holds except that the bound is loosened in this case because $\mathbf{H}$ always has full rank and $\hat{\mathbf{s}}=\mathbf{H}^{\dagger} \mathbf{r}$ is uniquely determined as required by the condition in (17). Therefore, we have the following corollary.

Corollary 4 . The relative estimation error of equalization in ZP-OFDM is bounded by

$$
\frac{\|\hat{\boldsymbol{s}}-\mathbf{s}\|}{\|\boldsymbol{s}\|} \equiv \frac{\left\|\mathbf{H}^{\dagger} \mathbf{r}-\mathbf{s}\right\|}{\|\boldsymbol{s}\|} \leq \frac{2 \kappa_{2}(\mathbf{H})}{\sqrt{\gamma_{\text {in }}}}+\mathcal{O}\left(\frac{1}{\gamma_{\text {in }}}\right) .
$$

Figure 5 shows the MSEE of ZF equalization in HIPERLAN/2 channel A and the upper bound from Corollary 4. We can see that the upper bound is not tight. This is due to a probable violation of the condition in (19). However, from Figure 5, we can see that this bound is proportional to the MSEE. Therefore, the bound acts as a prediction for the MSEE. We note that $\gamma_{\text {in }}$ may range from 0 to $20 \mathrm{~dB}$ in wireless communications and usually $\kappa_{2}(\mathbf{H}) \gg 1$. Therefore, $\mathcal{O}\left(1 / \gamma_{\text {in }}\right)$ is usually negligible in (20). If we define the SNR at the output of the equalizer $\gamma_{\text {out }} \stackrel{\text { def }}{=}\|\mathbf{s}\|^{2} /\|\hat{\boldsymbol{s}}-\mathbf{s}\|^{2} \equiv$ $\|\boldsymbol{s}\|^{2} /\left\|\mathbf{H}^{\dagger} \mathbf{s}-\mathbf{s}\right\|^{2}$ as in (12), we obtain approximately

$$
\gamma_{\text {out }} \geq \frac{\gamma_{\text {in }}}{4 \kappa_{2}^{2}(\mathbf{H})} .
$$

An example of the output SNR, $\gamma_{\text {out }}$, for ZF equalization in HIPERLAN/2 channel A is also shown in Figure 2 together with the bound from Corollary 4. Again, the bound gives a good prediction of $\gamma_{\text {out }}$.

Since system performance is determined by the SNR at the equalizer output $\gamma_{\text {out }}$ which can be predicted by $\kappa_{2}(\mathbf{H})$ together with $\gamma_{\text {in }}$, we may use $\kappa_{2}(\mathbf{H})$ as a system design criterion. To be specific, redundancy introduced by ZP can be reduced by means of inserting fewer zeros at the end of each block, which is referred to as AZP-OFDM, while keeping $\kappa_{2}(\mathbf{H})$ relatively low. Assuming $K$ zeros are padded, with $0 \leq K \leq L$, the new signal model is given by

$$
\mathbf{r}^{\prime}=\mathbf{H}^{\prime} \mathbf{s}_{N}+\boldsymbol{\eta}^{\prime},
$$

where $\mathbf{r}^{\prime}$ and $\boldsymbol{\eta}^{\prime}$ are $(N+K)$-vectors and $\mathbf{H}^{\prime}$ is a $(N+K) \times N$ matrix. Note that the received signal in AZP-OFDM (22) is just a "truncated" version of ZP-OFDM. The corresponding channel matrix $\mathbf{H}^{\prime}$ is a submatrix of the ZP-OFDM channel matrix $\mathbf{H}$ constructed by deleting its last $P-N-K$ rows. Let $\left\{\sigma_{i}\right\}_{i=1}^{N}$ denote the singular values of $\mathbf{H}$ and $\left\{\sigma_{i}^{\prime}\right\}_{i=1}^{N}$ denote the singular values of $\mathbf{H}^{\prime}$, both arranged in nonincreasing order. From the interlacing property of singular values [17], we know $\sigma_{1} \geq \sigma_{1}^{\prime} \geq \sigma_{2} \geq \sigma_{2}^{\prime} \geq \cdots \geq \sigma_{N} \geq \sigma_{N}^{\prime} \geq 0$. Usually $\sigma_{1} \gg \sigma_{N}$. Heuristically, $\sigma_{N}$ is more sensitive to perturbation. Therefore, deleting some of the last rows of $\mathbf{H}$, or in other words, padding with fewer zeros in ZP-OFDM increases $\kappa_{2}(\mathbf{H})$, as is confirmed by simulations. This is the price to pay for increased BW efficiency.

Of course, in so doing, the received signal will experience IBI, which can be canceled via decision feedback. Note that in AZP-OFDM, redundancy is reduced but still exists, which makes robust symbol recovery possible. Either ZF or MMSE equalization can be employed. Similar to ZP-OFDM, equalization in AZP-OFDM requires the solution of an overdetermined system of equations. The fast equalization algorithms developed in [10] cannot be applied due to their different structure of channel matrices. Therefore, AZP-OFDM trades off efficiency and performance, with the price of increased complexity.

\subsection{Modification for low-complexity equalization}

Alternatively, by taking advantage of the band-Toeplitz structure of the channel matrix, we propose a modification to the AZP-OFDM scheme. To be specific, if we discard $K$ initial observations, we obtain a system with the same number of observations as unknowns, that is,

$$
\mathbf{r}^{\prime \prime}=\mathbf{H}^{\prime \prime} \mathbf{s}+\boldsymbol{\eta}^{\prime \prime}
$$

where $\mathbf{r}^{\prime \prime}$ and $\boldsymbol{\eta}^{\prime \prime}$ are $N$-vectors and $\mathbf{H}^{\prime \prime}$ is an $N \times N$ Toeplitz matrix. We note that the received signal in (23) is a "windowed" version of that in ZP-OFDM (see (4)), with window width $N$ and delay $K$. As a result, ZF equalization is transformed into solving a band-Toeplitz system (with BW $L$ ) for which there are efficient solvers [18].

We note that the modified scheme differs from AZPOFDM in the number of trailing zeros $K$ (as will be discussed in Section 4.3) and in equalization at the receiver. Both the NGI and the proposed methods are windowed versions of ZP-OFDM with the same window width, but in the modified AZP scheme, the window is shifted to a "best" position to capture more the transmitted energy in the received signal, while in NGI-OFDM, the window is always fixed at the head part of the received block; in other words, the modified AZP system itself is nearly as overdetermined as in ZPOFDM (therefore, redundancy-based), but transformed for 


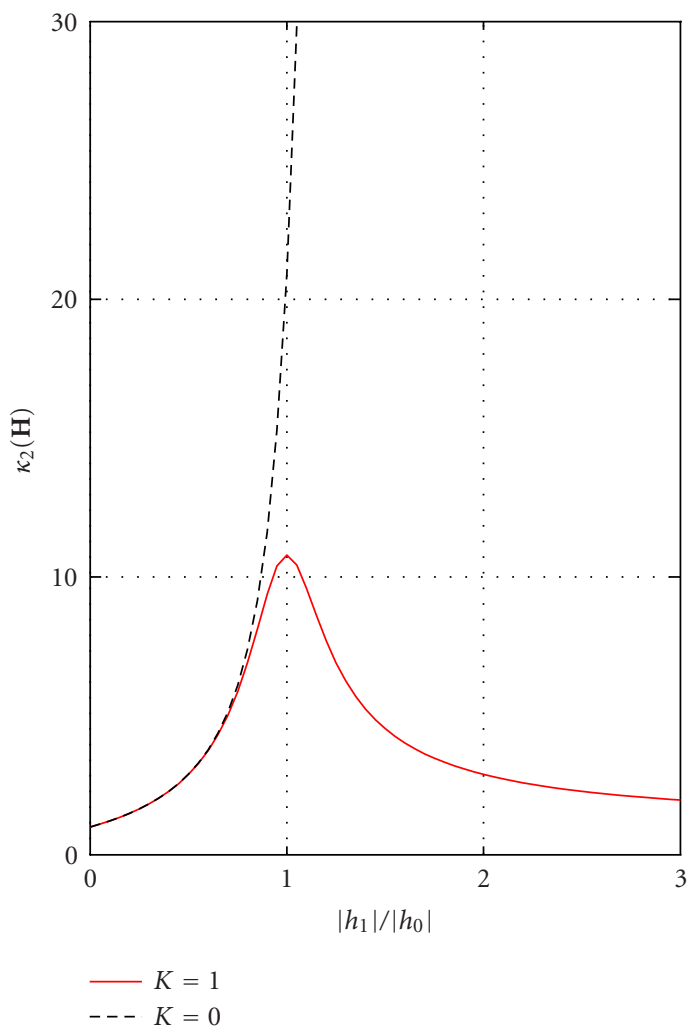

(a)

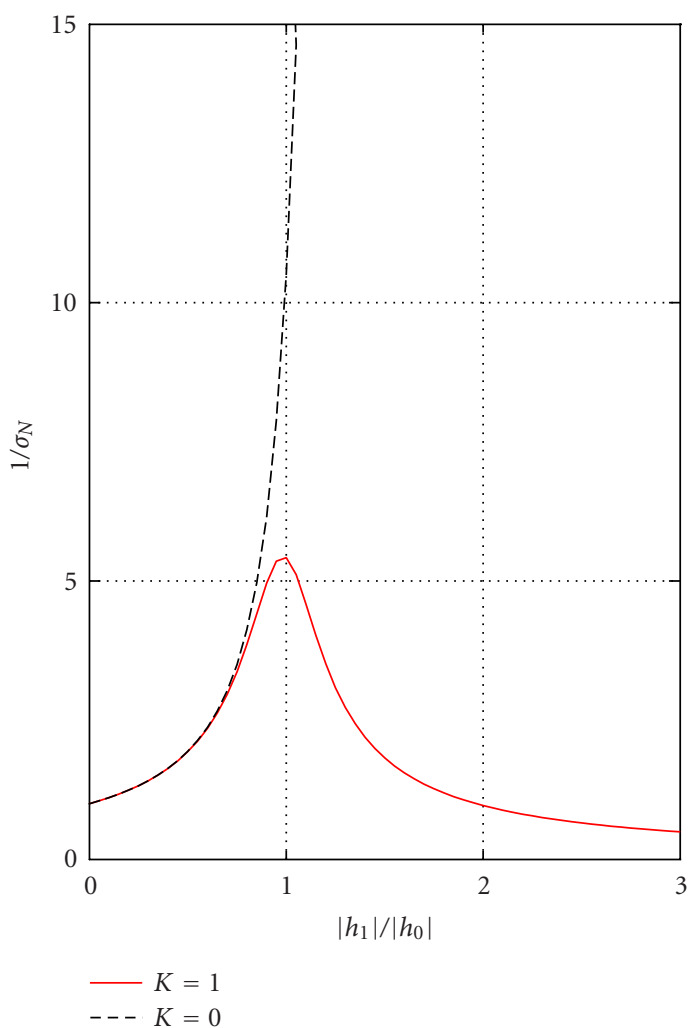

(b)

FIgURE 6: Criteria for choice of ZF length: $\kappa_{2}(\mathbf{H})$ versus $\sigma_{N}$ in two-tap channel.

simpler equalization, while NGI-OFDM does not introduce redundancy. Of course, by discarding some observations, we lose part of the redundancy in the received signal. As a result, the system performance will be slightly worse. However, due to the choice of AZP length (as will be clear in Section 4.3), the resulting system is still well-conditioned, as has been confirmed by our simulations.

\subsection{Choice of ZP length}

From the previous discussion, the condition number of the channel matrix can be used as a system design criterion. However, the calculation of the condition number of a matrix is computationally complex. Since $\kappa_{2}(\mathbf{H})=\sigma_{1} / \sigma_{N}$ and $\sigma_{N}$ is more sensitive to perturbation, $\kappa_{2}(\mathbf{H})$ depends mainly on $\sigma_{N}$. Hence, instead of using condition number, we consider the smallest singular value of the channel matrix, $\sigma_{N}$, as a criterion. In (20), if we substitute $\kappa_{2}(\mathbf{H})$ by $1 / \sigma_{N}$, we obtain an approximation bound for MSEE and $\gamma_{\text {out }}$, respectively, as

$$
\begin{gathered}
\frac{\|\hat{\mathbf{s}}-\mathbf{s}\|}{\|\mathbf{s}\|} \lesssim \frac{2}{\sigma_{N} \sqrt{\gamma_{\text {in }}}}+\mathcal{O}\left(\frac{1}{\gamma_{\text {in }}}\right), \\
\gamma_{\text {out }} \geqq \frac{1}{4} \gamma_{\text {in }} \sigma_{N}^{2} .
\end{gathered}
$$

To gain more insight into the criteria for choice of $\mathrm{ZP}$ length, we consider a special case of a two-tap channel, that
TABLE 1: Inverse iteration of power method.

$$
\begin{aligned}
& \text { Initialization: choose } \mathbf{x}_{0} ; i=0 . \\
& \text { Iteration: until convergence, } \\
& \qquad \begin{array}{ll}
\mathbf{y}_{i+1}=(\mathbf{A}-\sigma \mathbf{I})^{-1} \mathbf{x}_{i} & \\
\mathbf{x}_{i+1}=\mathbf{y}_{i+1} /\left\|\mathbf{y}_{i+1}\right\|_{2} & \text { (approx. eigenvector) } \\
\lambda_{i+1}=\mathbf{x}_{i+1}^{H} \mathbf{A} \mathbf{x}_{i+1} & \text { (approx. eigenvalue) } \\
i=i+1 &
\end{array}
\end{aligned}
$$

is, $\mathbf{h}=\left[h_{0}, h_{1}, 0, \ldots, 0\right]^{T}$. For this channel, the ZP length $K \in\{0,1\}$. It can be shown that singular values of channel matrices (of both $K=0$ and 1) depend only on $\left|h_{0}\right|$ and $\left|h_{1}\right|$. Figure 6 compares two criteria for choice of ZP length in all possible two-tap channels. We see that $1 / \sigma_{N}$ offers a close approximation to $\kappa_{2}(\mathbf{H})$, up to a scaling factor. An example of the approximate bound for the MSEE is also shown in Figure 5. From Figure 5, we see that (24) can also be used to predict the MSEE.

Fortunately, some efficient algorithms have been developed for calculating the smallest singular value of a matrix [13]. Here we adopt the power method, which can find the largest magnitude eigenvalue of a matrix $\mathbf{A}$ and the corresponding eigenvector. To find the other eigenpairs, the power method can be applied to $(\mathbf{A}-\sigma \mathbf{I})^{-1}$ for some shift $\sigma$, and it will converge to the eigenvalue closest to $\sigma$. By choosing $\sigma$ 


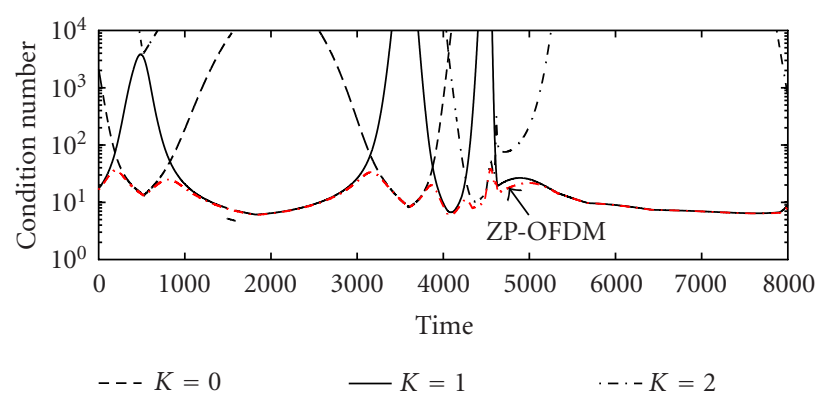

(a)

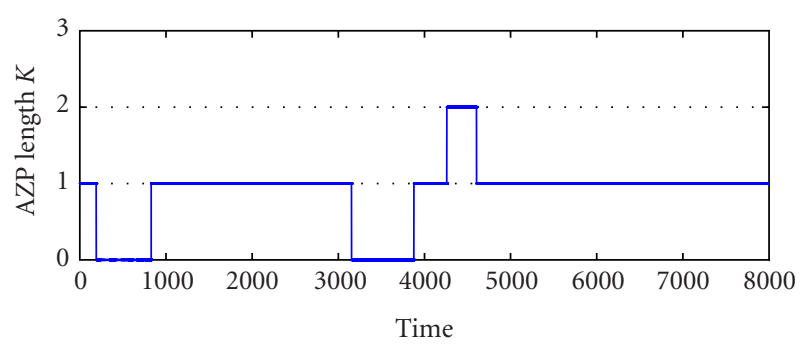

(b)

FIGURE 7: Variation of AZP lengths with time for HIPERLAN/2 channel model A with terminal speed $v=3 \mathrm{~m} / \mathrm{s}$ : (a) shows condition number of channel matrix for different AZP lengths while (b) presents adaptively selected AZP length versus time.

very close to a desired eigenvalue, convergence of the algorithm can be accelerated [13]. Table 1 details the algorithm. For a complete treatment of the power method and its variations, see $[12,13]$.

To estimate the smallest singular value of $\mathbf{H}$, the power method can be applied to $\left(\mathbf{H}^{H} \mathbf{H}-\sigma^{2} \mathbf{I}\right)^{-1}$ with an initial shift $\sigma$ equals 0 or previous estimate. The initial guess of the singular value can be chosen to be the previous estimate as well due to the slowly changing property of the channel matrix spectra as discussed in Section 5. Specifically, for the modified AZP-OFDM, to take the advantage of the channel matrix structure, we set $\sigma=0$ so that in every iteration, the computational cost is mainly solving two band-Toeplitz systems, that is, $\left(\mathbf{H}^{H} \mathbf{H}\right)^{-1}=$ $\mathbf{H}^{-1} \mathbf{H}^{-H}$. The convergence criterion for the power method used in our proposed method can be set quite loose because we are not required to estimate the singular value accurately.

Remark 1. We note that the choice of the number of padded zeros of modified AZP-OFDM may be different from that of the original AZP-OFDM. For the original AZP scheme, the condition number of the channel matrix will decrease with an increase of AZP length. Therefore, the singular-valuefinding algorithm may stop whenever the smallest singular value is large enough to guarantee symbol recovery. Beyond that, increasing AZP length will improve system performance, but the BW efficiency will decrease, and therefore its choice may depend on the system requirements. On the other hand, in the modified scheme, there exists some optimal choice of AZP length. Heuristically, if we slide a window on the received block, there exists an optimal shift for the windowed sequence to capture most of the energy in the original sequence. This can be achieved by a simple search procedure.

Since wireless channels are well modelled as stochastic processes, the system parameter $K$ is random. However, it is difficult to find a closed-form expression for statistics of $K$. In Section 5, we will instead characterize $K$ by Monte-Carlo simulations.

\section{SIMULATION RESULTS AND DISCUSSIONS}

\subsection{Statistics of AZP length}

Standard HIPERLAN/2 channel models [15] are used. The OFDM block size of 64 and a terminal speed of $3 \mathrm{~m} / \mathrm{s}$ are assumed. The expected BW efficiency for different channel models ranges from 93\%-100\%, while employing CP- or ZPOFDM, BW efficiency is fixed at $80 \%$. The variation of AZP length depends on the velocity of the mobile terminal. An example of the variation of the channel matrix condition number with different numbers of padding zeros as well as variation of the estimated $K$ with time is shown in Figure 7 for channel A with a terminal speed of $3 \mathrm{~m} / \mathrm{s}$. We see that for a terminal speed of $3 \mathrm{~m} / \mathrm{s}$, the AZP length varies very slowly and it most likely changes among neighboring values. This is due to small changes in the channel only resulting in small variations of the spectra of the channel matrix [17].

\subsection{Complexity issues}

The AZP length results lead to further reductions in implementation complexity in that the AZP length need not be updated very frequently. The search algorithm can be simplified, examining several neighboring values, instead of performing exhaustive search over the set of all possible values. Moreover, since in the proposed schemes, accurate estimate of the smallest singular value is not required, the convergence criterion in applying the power method can be very loose, which will reduce the number of iterations significantly.

Equalization in (22) is a band-Toeplitz LS problem, for which special methods have been devised of complexity $O((N+K) N)$. Some "superfast" methods require only $O((N+K) \log N)$ operations [19]. For modified AZP-OFDM, in each iteration for choosing AZP length and in the processing of each data block, the main cost is for solving bandToeplitz equations. Usually the system design results in a narrowband matrix. As a result, the computational cost can be significantly reduced [12]. The complexity of CP-OFDM is $O(N \log N)$, while using ZP-OFDM with fast equalization algorithms [10], the complexity is $O(P \log P)$. In our simulations under the context of HIPERLAN/2 channel models, we 


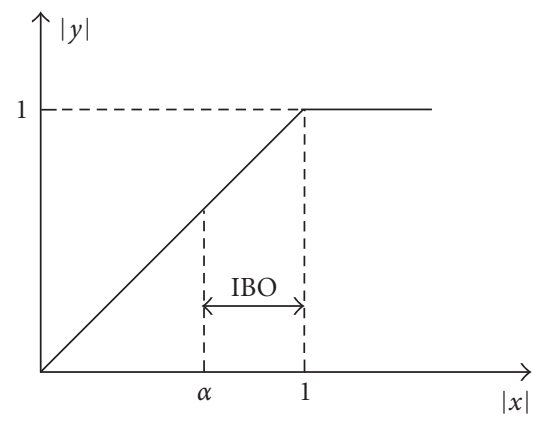

Figure 8: Simplified PA model.

estimate AZP length every 20 blocks and the power method usually converges in several iterations. In summary, the proposed scheme has almost the same complexity as ZP-OFDM, both of which are slightly higher than that of CP-OFDM.

\subsection{Clipping effects}

As is well known, one of the major drawbacks of OFDM is the high PAPR of the signal to be transmitted. As a result, OFDM signals cause serious problems such as distortion of the transmitted signal due to the PA nonlinearity. In this paper, we consider the simplified PA model shown in Figure 8, with both the input and output normalized as in [10]. According to [10], by defining the clipping ratio $\mathcal{C}$ as the number of clipped symbols over the total number of symbols, and the IBO as the ratio of the mean power at PA input to the input saturation power, (which is a function of $\mathcal{C}$ ), the transmitter output SNR difference between CP- and ZP-OFDM is given by [10]

$$
\Delta_{\text {zp }}(\mathcal{C})=10 \log \left(\frac{P}{N}\right)+\mathrm{IBO}_{\mathrm{zp}}(\mathcal{C})-\mathrm{IBO}_{\mathrm{cp}}(\mathcal{C}) .
$$

Therefore, such SNR loss will be smaller with a shorter guard interval as is the case in our proposed AZP-OFDM. Since the length of AZP, $K$, is not fixed, we can estimate the expected SNR loss compared with CP-OFDM as follows:

$$
\begin{aligned}
& \overline{\Delta_{\text {azp }}}(\mathcal{C}) \\
& =E_{K}\left[10 \log \frac{N+K}{N}+\mathrm{IBO}_{\text {azp }}(\mathcal{C})-\mathrm{IBO}_{\mathrm{cp}}(\mathcal{C})\right] .
\end{aligned}
$$

For HIPERLAN/2 transmission, Figure 9 shows the simulated SNR loss for ZP- and AZP-OFDM with respect to CPOFDM. The clipping effect alone requires reducing the transmit power by less than $0.3 \mathrm{~dB}$ compared to CP-OFDM in order to guarantee the same amount of out-of-band radiation, while the reduction for ZP-OFDM is about $0.9 \mathrm{~dB}$ [10].

\subsection{Uncoded BER performance}

Example 1 (uncoded BER in HIPERLAN/2 channel A). Figure 10 shows the uncoded BER performances of NGI-, CP-, ZP-, and the proposed modified AZP-OFDM scheme in channel A. The AZP length $K(n)$ is updated every 20 blocks. NGI-OFDM experiences serious error floors. AZP-

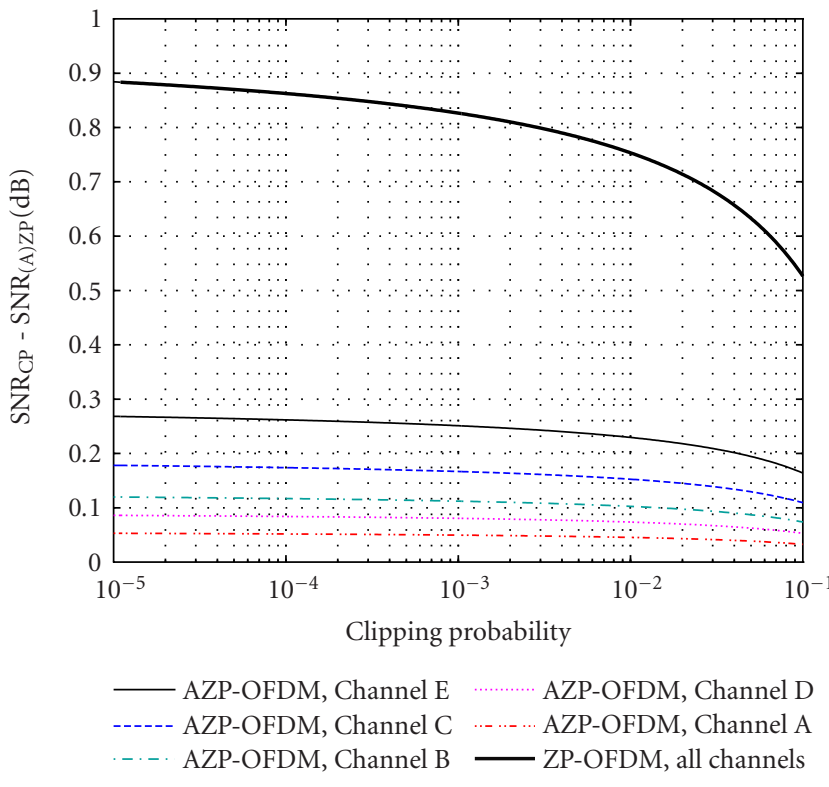

Figure 9: SNR difference between CP and (A)ZP induced by clipping effects.

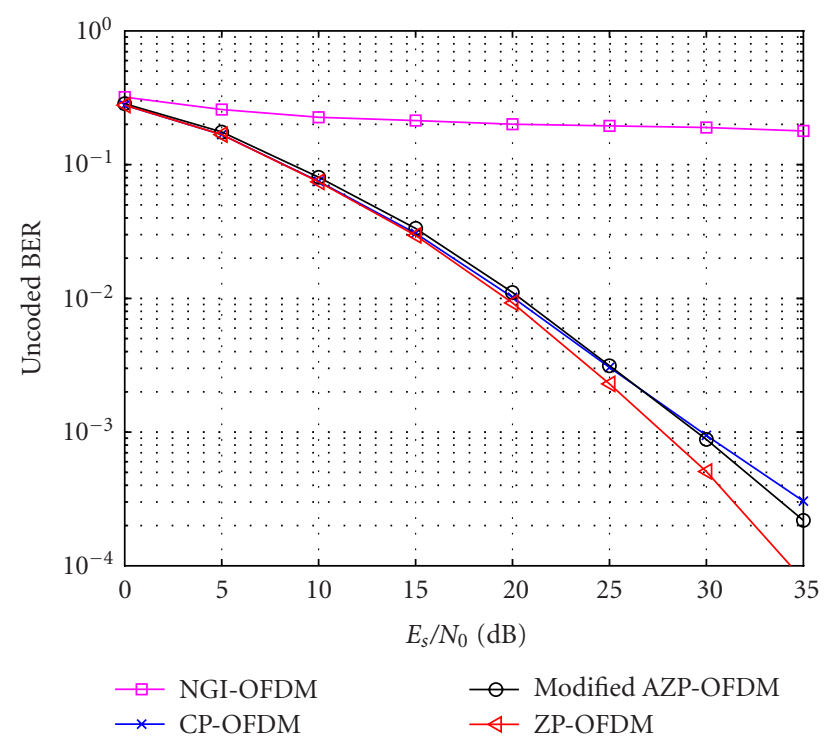

Figure 10: Uncoded BER in HIPERLAN/2 channel A for QPSK.

OFDM has similar performance to CP-OFDM at low SNR, and both are slightly worse than that of ZP-OFDM. This is due to the guaranteed symbol recovery capability in $\mathrm{ZP}$ OFDM, whereas for AZP-OFDM, this property has been sacrificed to achieve higher BW efficiency and lower complexity.

Example 2 (uncoded BER in channels with exponential power delay profile). We assume channel model order $L=$ $N$, and channel tap powers $\rho_{l}^{2}=\beta e^{-\alpha l}$, for $l=0,1, \ldots, N-1$, with $\beta$ chosen to normalize the total channel power, that is, $\beta=1 / \sum_{l=0}^{N-1} e^{-\alpha l}$ and $\sum_{l=0}^{N-1} \rho_{l}^{2}=1$. Obviously, small $\alpha$ 


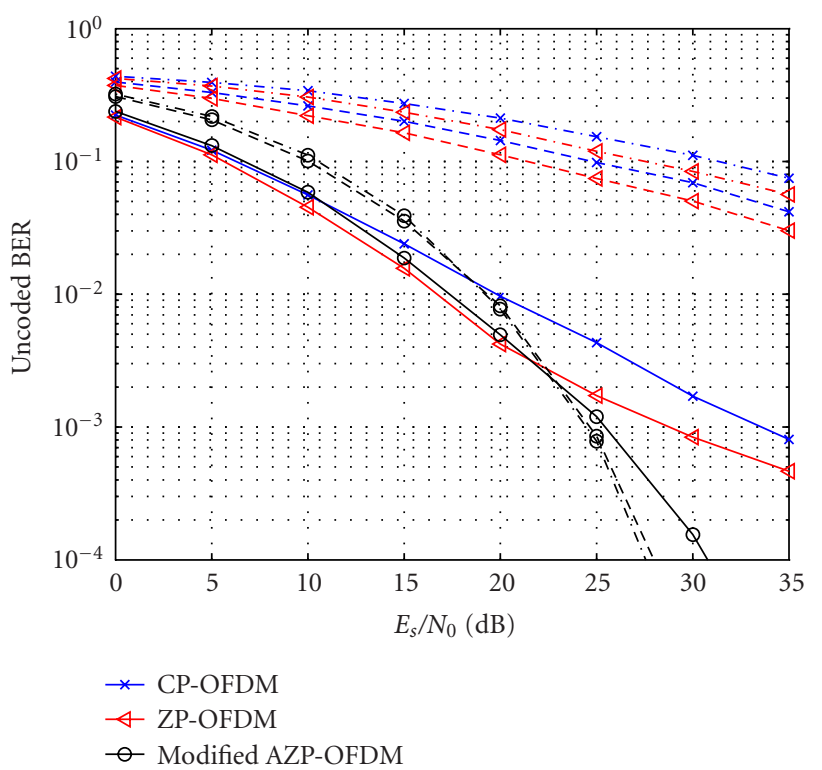

FIGURE 11: Uncoded BER in channels with exponential power delay profiles for QPSK, $N=32, G=4$, (solid line: $\alpha=0.5$, dashed line: $\alpha=0.1$, dashed-dotted line: $\alpha=0.05$ ).

corresponds to large rms delay spread. Figure 11 shows the uncoded BER performance of CP-OFDM, ZP-OFDM, and AZP-OFDM in a system with block size $N=32$ and guard length $G=4$. We see that for small $\alpha$, both CP- and ZPOFDM, with fixed guard length, experience serious error floors. Using AZP-OFDM, since the guard length is adaptive, the system is more robust to channels with large delay spread. The expected BW efficiencies of AZP-OFDM for different $\alpha$ are, respectively, $71.35 \%(\alpha=0.05), 77.20 \%(\alpha=0.1)$, and $90.27 \%(\alpha=0.5)$, compared with fixed BW efficiencies in CP- and ZP-OFDM, 88.89\%. In other words, when the delay spread is low, AZP-OFDM has performance slightly below that of CP- and ZP-OFDM, while if the delay spread is large, performance can be maintained at the expense of BW efficiency.

\section{CONCLUSIONS}

This paper has shown how to optimize the transmit redundancy for OFDM transmission over wireless channels to tradeoff performance, complexity, and BW efficiency. A novel BW efficient zero-padding scheme, AZP-OFDM, was introduced based on the channel matrix condition, which is flexible for system design compared with the standard CPIZP-OFDM. The new AZP-OFDM transmission method offers robust performance over a large range of time dispersion (delay spread) while keeping implementation complexity low.

\section{ACKNOWLEDGMENT}

This research was supported in part by Natural Sciences and Engineering Research Council CRD Project Grant 22687699.

\section{REFERENCES}

[1] Z. Wang and G. B. Giannakis, "Wireless multicarrier communications," IEEE Signal Processing Magazine, vol. 17, no. 3, pp. 29-48, 2000.

[2] A. Scaglione, G. B. Giannakis, and S. Barbarossa, "Redundant filterbank precoders and equalizers. I. Unification and optimal designs," IEEE Trans. Signal Processing, vol. 47, no. 7, pp. 1988-2006, 1999.

[3] M. Toeltsch and A. F. Molisch, "Efficient OFDM transmission without cyclic prefix over frequency-selective channels," in Proc. 11th IEEE International Symposium on Personal, Indoor and Mobile Radio Communications (PIMRC '00), vol. 2, pp. 1363-1367, London, UK, September 2000.

[4] V. Vadde and S. Gray, "Partial response signaling for enhanced spectral efficiency and RF performance in OFDM systems," in Proc. IEEE Global Telecommunications Conference (GLOBECOM '01), vol. 5, pp. 3120-3124, San Antonio, Tex, USA, November 2001.

[5] X.-G. Xia, "Precoded and vector OFDM robust to channel spectral nulls and with reduced cyclic prefix length in single transmit antenna systems," IEEE Trans. Communications, vol. 49, no. 8, pp. 1363-1374, 2001.

[6] P. J. W. Melsa, R. C. Younce, and C. E. Rohrs, "Impulse response shortening for discrete multitone transceivers," IEEE Trans. Communications, vol. 44, no. 12, pp. 1662-1672, 1996.

[7] H. Ochiai and H. Imai, "On the distribution of the peak-toaverage power ratio in OFDM signals," IEEE Trans. Communications, vol. 49, no. 2, pp. 282-289, 2001.

[8] D. Kim and G. L. Stüber, "Residual ISI cancellation for OFDM with applications to HDTV broadcasting," IEEE Journal on Selected Areas in Communications, vol. 16, no. 8, pp. 15901599, 1998.

[9] G. H. Golub and C. F. van Loan, Matrix Computations, Johns Hopkins University Press, Baltimore, Md, USA, 2nd edition, 1989.

[10] B. Muquet, Z. Wang, G. B. Giannakis, M. de Courville, and P. Duhamel, "Cyclic prefixing or zero padding for wireless multicarrier transmissions?," IEEE Trans. Communications, vol. 50, no. 12, pp. 2136-2148, 2002.

[11] E. C. Ifeachor and B. W. Jervis, Digital Signal Processing: A Practical Approach, Prentice-Hall, Englewood Cliffs, NJ, USA, 2nd edition, 2002.

[12] G. Dahlquist and Å. Björck, Numerical Mathematics and Scientific Computation. Vol. 2, SIAM, Philadelphia, Pa, USA, 2003.

[13] J. W. Demmel, Applied Numerical Linear Algebra, SIAM, Philadelphia, Pa, USA, 1997.

[14] J. G. Proakis, Digital Communications, McGraw-Hill, New York, NY, USA, 3rd edition, 1995.

[15] ETSI Normalization Committee, "Channel models for HIPERLAN/2 in different indoor scenarios," Norme ETSI, document 3ERI085B, Sophia-Antipolis, Valbonne, France, 1998.

[16] S. K. Mitra, Digital Signal Processing: A Computer Based Approach, McGraw-Hill, New York, NY, USA, 2nd edition, 2001.

[17] R. A. Horn and C. R. Johnson, Matrix Analysis, Cambridge University Press, New York, NY, USA, 1994.

[18] S. Chandrasekaran and A. H. Sayed, "A fast stable solver for nonsymmetric toeplitz and quasi-toeplitz systems of linear equations," SIAM Journal on Matrix Analysis and Applications, vol. 19, no. 1, pp. 107-139, 1998.

[19] Å. Björck, Numerical Methods for Least Squares Problems, SIAM, Philadelphia, Pa, USA, 1996. 
Neng Wang received his B.E. degree in electrical engineering from Shanghai Jiao Tong University, Shanghai, China, in 1994, and the M.E. degree in electrical engineering from Nanjing University of Posts and Telecommunications, Nanjing, China, in 1999. Since 2000, he has been working towards the Ph.D. degree and serving as a Research Assistant in the Department of Electrical and Computer Engineering, Queen's

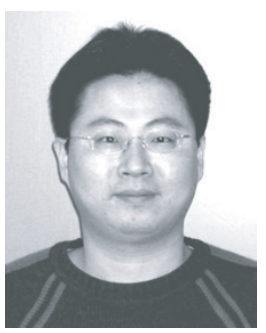
University, Kingston, Ontario, Canada. He was an Electronics Engineer in Suzhou Lambeau High-Tech Enterprise Group, Suzhou, China, from 1994 to 1996. His research interests lie in wireless multicarrier and multiple-input multiple-output (MIMO) communication systems, and general areas of wireless communications and signal processing.

Steven D. Blostein received his B.S. degree in electrical engineering from Cornell University in 1983, and the M.S. and Ph.D. degrees in electrical and computer engineering from the University of Illinois at Urbana-Champaign, in 1985 and 1988, respectively. He has been on the faculty at Queen's University in Kingston, Ontario, Canada, since 1988, where he currently holds the position of Professor and Head of

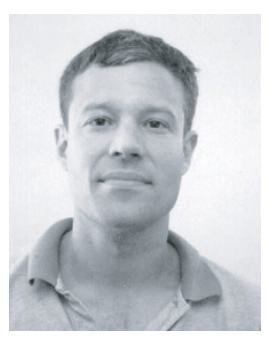
the Department of Electrical and Computer Engineering. His current interests lie in signal processing for wireless communications as well as detection and estimation theory. He is a Senior Member of IEEE and a registered Professional Engineer in Ontario. 